Bull. Austral. Math. Soc.

VOL. 38 (1988) [479-480]

\title{
THE DIRICHLET PROBLEM FOR DEGENERATE ELLIPTIC 2-DIMENSIONAL MONGE-AMPËE EQUATION: CORRIGENDUM
}

\author{
Kazuo Amano
}

The author would like to make several minor amendments to [1]. page 390

(1) 1.25 Replace $C^{s *+2 d}$ by $C^{s *+2, d}$.

(2) (2.6) Replace $W$ by a small $w$.

(3) (2.22) The correct inequality is " $\left\|v_{n}\right\|_{s} \leqslant \ldots$ ".

(4) (2.28) The number $s$ should be less than or equal to $s_{*}-2$.

page 397

(5) 1.2 Insert an inequality " $\leqslant$ " between $\left\|v_{0}\right\|_{\text {s }}$ and $\sqrt{\varepsilon}$.

(6) (2.52) The correct inequality is " $\left\|v_{n}\right\|_{0} \leqslant K\left\{\left\|f_{n}\right\|_{0}+\cdots\right\}$ ".

page 401

(7) 1.6 Replace $\Lambda_{k}$ by $A_{k}$.

page 402

(8) 1.7 Replace $\left(T_{e}^{t \Phi}\right)^{-1}$ by $\left(T-e^{T \Phi}\right)^{-1}$.

(9) 1.20 Use a small $c(t ;$.$) instead of a capital C(t ;$.$) .$

page 403

(10) 1.11 The number $i$ should be less than or equal to $N$.

(11) (3.16) Replace $i+h$ by $i+j$.

page 408

(12) 1.3-1.16 Replace all $s^{*}$ by $s_{*}$.

page 409

(13) 1.4 The correct expression should be " $\cdots+\varepsilon_{n}\left(\alpha^{i j} \bar{v}_{i} \bar{v}_{j}\right) \ldots$ ".

Received 29th July 1988.

Copyright Clearance Centre, Inc. Serial-fee code: 0004-9729/88 \$A2.00+0.00. 


\section{REFERENCES}

[1] K. Amano, 'The Dirichlet problem for degenerate elliptic 2-dimensional Monge-Ampère equation' Bull A ustral. Math. Soc. 38 (1988), 389-410.

\section{Department of Mathematics}

Josai University

1-1 Keyakidai

Sakado, Saitama 350-02

Japan 